\title{
Citation Index
}

\begin{tabular}{|c|c|}
\hline Pentateuch & -Shabbat \\
\hline -Genesis & $4 a 98 \mathrm{n} 31$ \\
\hline $1: 2869$ & $5 a 128 \mathrm{n} 177$ \\
\hline $4: 270$ & $7 a 103$ \\
\hline $4: 2570$ & $18 b$ 117n134 \\
\hline -Exodus & $28 a 116 n 133$ \\
\hline $25: 2724 n 85$ & $28 b$ 128n177 \\
\hline -Leviticus & $31 a 130$ \\
\hline $4: 26142,143$ & $37 b$ 128n177 \\
\hline $4: 28142$ & $67 a 103$ \\
\hline $19: 1926$ & $69 a$ 117n134 \\
\hline -Deuteronomy & $71 b$ 141-144 \\
\hline \multirow[t]{2}{*}{$22: 1126$} & $73 a 128 \mathrm{n} 177$ \\
\hline & $93 b 99 \mathrm{n} 36$ \\
\hline Prophets & $102 b \quad 128 \mathrm{n} 177$ \\
\hline -Jeremiah & $111 b 103$ \\
\hline \multirow[t]{2}{*}{$23: 28183$} & $112 a 152$ \\
\hline & $129 b$ 128n177 \\
\hline Writings & $138 a 104 \mathrm{n} 71$ \\
\hline -Psalms & $142 a 99 \mathrm{n} 35$ \\
\hline 119:126 32 & $157 a$ 176n183 \\
\hline -Esther & -Eruvin \\
\hline $2: 2270$ & $28 a 98 \mathrm{n} 32$ \\
\hline -I Chronicles & $32 b$ 95n18, 97 \\
\hline \multirow[t]{2}{*}{$4: 943$} & $63 b-64 a 103 n 62$ \\
\hline & $90 a 145 \mathrm{n} 59$ \\
\hline Mishnah & -Pesahim \\
\hline- Avot & $3 b 124 \mathrm{n} 167$ \\
\hline $1: 4106$ & $7 a 99 \mathrm{n} 36$ \\
\hline $6: 170 \mathrm{n} 27$ & $11 b-12 a 103 n 62$ \\
\hline -Bava Metz’ia & $74 b 177$ \\
\hline $4: 1115$ & $78 b$ 117n134, 117n135 \\
\hline -Sanhedrin & $81 b 119$ \\
\hline \multirow[t]{2}{*}{$10: 17 n 3$} & $101 b 157$ \\
\hline & $105 b$ 108-109 \\
\hline Talmud Bavli & -Yoma \\
\hline -Berakhot & $10 b 117 \mathrm{n} 134$ \\
\hline $20 a 102 \mathrm{n} 57$ & $16 b 145 \mathrm{n} 59$ \\
\hline $21 a 127$ & $28 b 34$ \\
\hline $27 a 117 n 134$ & $69 a \quad 26,27$ \\
\hline $44 a 99 n 35$ & $78 a 132,135$ \\
\hline $55 a 183$ & $79 a 132 \mathrm{n} 3$ \\
\hline $59 a-b$ 109n97 & 8043 \\
\hline
\end{tabular}

Ә Open Access. (C) 2021 Ari Bergmann, published by De Gruyter. (c) BY-NC-ND This work is licensed under a Creative Commons Attribution-NonCommercial-NoDerivatives 4.0 International License. https://doi.org/10.1515/9783110709834-009 


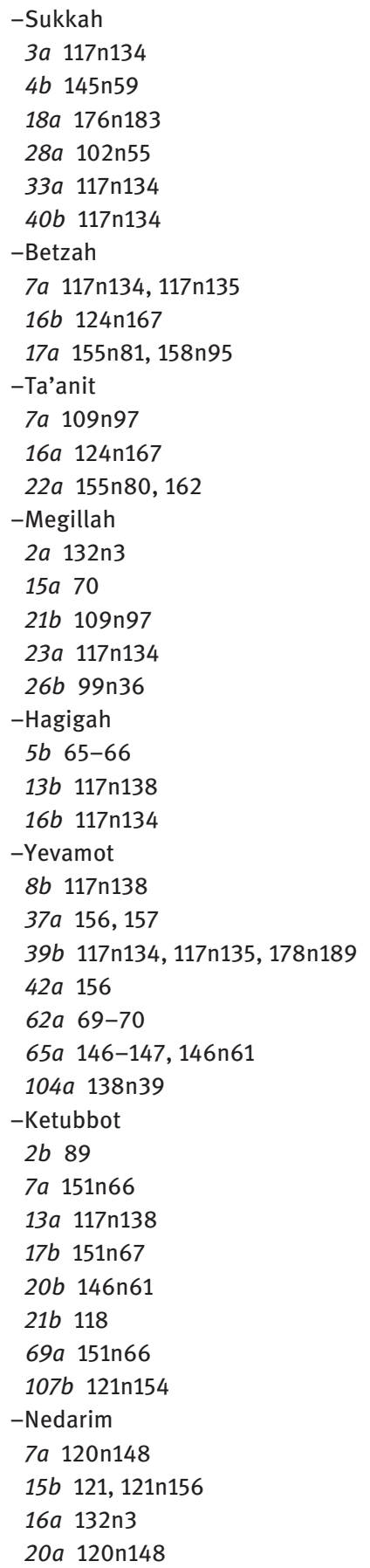

$27 a$ 117n134

$32 b$ 121n156

$33 b$ 121n154

$60 b \quad 135$

$82 b \quad 121 n 154$

90a 135

- Nazir

12a 120n148

19a 117n134, 117n135

20a 120n148

22a $120 \mathrm{n} 148$

-Sotah

$8 b 146$

20a $154 \mathrm{n} 77$

25a $178 \mathrm{n} 189$

40a 109 n97

$44 b$ 117n134

-Gittin

$\begin{array}{lll}6 b & 154 \mathrm{n} 77\end{array}$

$39 b$ 99n35

$62 b 118$

-Qiddushin 79n75, 168, 172, 175

3b 164

6b $116 \mathrm{n} 133$

$42 a 98 \mathrm{n} 31$

$73 a 109$

58a-b 121n154

-Bava Qamma

$86 a$ 117n134

90a 116-117, 116n133, 117n134, $117 \mathrm{n} 135$

$93 b 104,104 n 68$

-Bava Metzi’a

2a 180n194

3a 180n194

5a 180n194

$7 b$ 180n194

$10 b 135$

$12 a$ 180n194

13a-b 180n194

14a 180n194

$15 b$ 180n194

$19 a-b$ 180n194

$26 b$ 180n194

28 a 180n194

38a 180n194

$44 a 115$ 


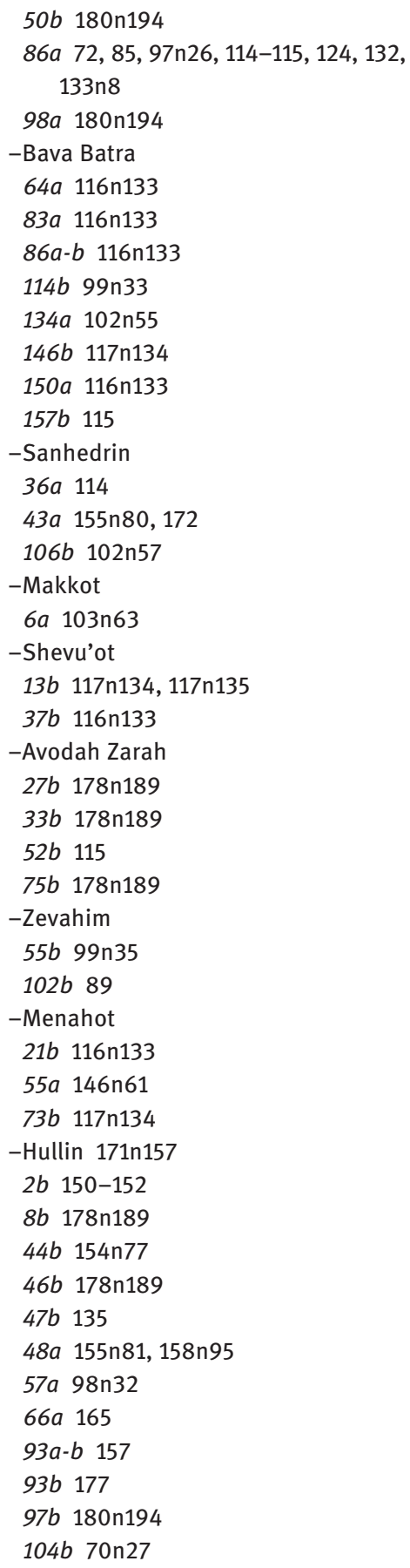

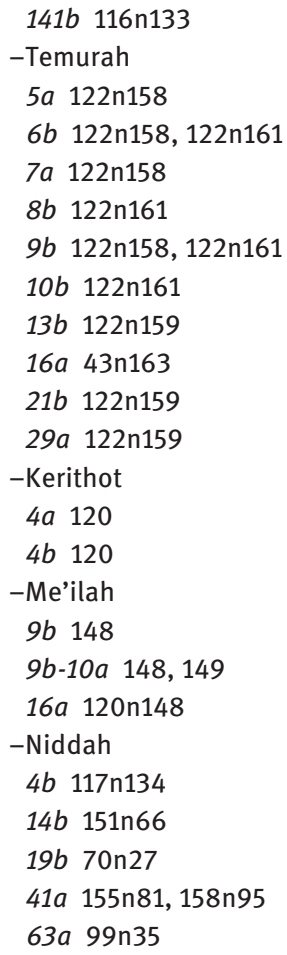

\section{Rishonim}

-Maimonides (Rambam) to m.Sanhedrin 10:1 7n3 Mishneh Torah Introduction 44n166, 85 1:1-4 85n112 Kil'ayim [Prohibited Mixtures] 10:32 26n91

Mamrim [Rebels] 1:1-2 191n39 2:1 $35 \mathrm{n} 126$ 2:2-3 43n164 3:4 191n39

Sanhedrin 5:1 191n38

Sefer hamitsvot Principle 2, 35n126

-Nahmanides (Ramban)

Sefer hazekhut to the Rif on 


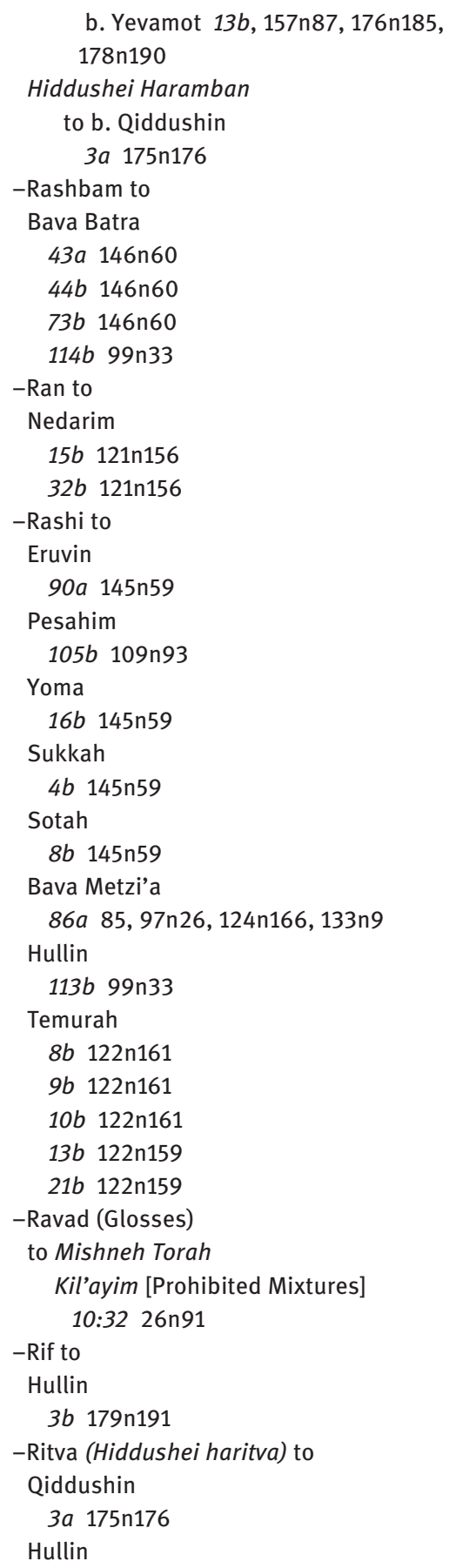
178n190

Hiddushei Haramban to b. Qiddushin 3a 175 n176

-Rashbam to

Bava Batra

43a $146 \mathrm{n} 60$

$44 b 146 \mathrm{n} 60$

$73 b \quad 146 n 60$

$114 b$ 99n33

- Ran to

Nedarim

$15 b$ 121n156

$32 b \quad 121 n 156$

-Rashi to

Eruvin

90a $145 \mathrm{n} 59$

Pesahim

105b 109n93

Yoma

$16 b 145 \mathrm{n} 59$

Sukkah

$4 b \quad 145 n 59$

Sotah

$8 b \quad 145 n 59$

Bava Metzi'a

$86 a 85,97 n 26,124 n 166,133 n 9$

Hullin

$113 b$ 99n33

Temurah

$8 b \quad 122 \mathrm{n} 161$

$9 b \quad 122 \mathrm{n} 161$

10b $122 \mathrm{n} 161$

$13 b \quad 122 n 159$

21b 122n159

-Ravad (Glosses)

to Mishneh Torah

Kil'ayim [Prohibited Mixtures]

10:32 26n91

-Rif to

Hullin

3b 179n191

-Ritva (Hiddushei haritva) to

Qiddushin

3a $175 \mathrm{n} 176$

Hullin

97b 180n194

-Rosh to

Shabbat

$9 b$ 140n49

Nedarim

2b 120 n148

Bava Metzi'a

$16 b$ 140n49

Sanhedrin

4:6 112n112

-Tosafot to

Shabbat

9b 140 n49

Pesahim

81b 119n144

Yoma

$40 b 146 \mathrm{n} 60$

Ketubbot

$2 b$ 176n180

Nedarim

7a 120n148

20a 120n148

Nazir

12a 120n148

20a 120n148

22a 120 n148

Sotah

$38 a$ 146n60

Bava Qamma

93b 104n68, 104n69

Bava Batra

$175 b$ 146n60

Zevahim

$102 b$ 176n180

Menahot

58b 118-119n142

Hullin

$2 b$ 112n112, 140n49

48 a $158 n 95$

Me'ilah

16a 120n148

Temurah

29a 122n159

-Zerahia Halevy of Girona (Ba'al Hama'or) Hama'or haqatan, on the Rif to

b. Pesahim 20a, 157n 86

b. Pesahim 101b, 157 


\section{Aharonim}

-Bi'ur hagra (Gaon Rav Eliyahu of Vilna) to

Orakh Haim (Shulhan arukh)

443:1 98n31

-Bi'ur hagrah al hashas (Hayyim

Soloveitchik) to

b. Yoma

$69 a$ 27n96

-Gilyon hashas (Akiva Eiger) to

b. Hullin

93b $178 \mathrm{n} 188$

-Hiddushei Anshei Shem to

b. Hullin

3b 179n191

-Kesef Mishneh (Joseph Caro) to

Mishneh Torah (Maimonides)

Laws of Impermissible Mixtures [Kil'ayim] (re: Yoma 69a)

10:32 27n92

-Kitsur kelalei hatalmud (Yehudah Aryeh

Leib of Frankfurt) to

b. Berakhot (Rule 7), 100n40

-Mitspeh Eitan (Abraham ben Judah Leib

Maskileison) to

b. Horayot

14a 105n75
-Sha'agat Aryeh (Aryeh Leib Gunzberg) to Mishneh Torah (Maimonides)

Laws of Impermissible Mixtures [Kil'ayim] (re: Yoma 69a)

10:32 (178) 27n92

-Shittah mequbetset (Betzalel ben Avraham Ashkenzazi) to

b. Betzah

17a 158 n95

b. Bava Metzi'a

2a $180 \mathrm{n} 194$

3a 180n194

5a $180 \mathrm{n} 194$

$7 b$ 180n194

$12 a$ 180n194

$13 a-b$ 180n194

$14 a$ 180n194

$15 b$ 180n194

26 b 180n194

28a 180n194

38 a 180n194

$50 b$ 180n194

98a 180n194

b. Nazir

15a 120n148

$18 b \quad 120 n 148$ 
